Results During the study period 770 newborns were hospitalized in NICU due to various pathological conditions and 50 newborns have been selected with AKI. As the control group, 50 newborns were taken with comparable associated pathological conditions, but without kidney injury. The calculated prevalence of AKI in neonates was 6.4\%. Most of involved neonates in the study in both groups (AKI and non AKI) were born at term $(64 \%$ and $54 \%)$ with predominance of male $(68 \%$ and $60 \%)$. The mortality rate was higher in newborns with AKI than control group (36\% vs $24 \%$ ). In half of newborn infants with AKI predominate severe score level, while in control group predominate median score level (42\%). There is a significant difference between the mean score value in neonates with AKI and lethal outcome compared to neonates with AKI without lethal outcome (70.73 \pm 18.6 vs. $40.2 \pm 16.6)$.

Conclusion Acute kidney injury is a life threatening condition with still high mortality rate. The severity of the illness of hospitalized newborn infants in NICU is estimated by SNAPPE 2 score. The high score level is associated with the severity of the disease and higher mortality. Appropriate treatment of newborns with severe kidney injury improves the outcome and reduces the mortality of the disease.

\section{P632 MORBIDITY AND MORTALITY OUTCOMES IN NEONATES WHO WERE TRANSFERRED FROM TO THE NEONATAL INTENSIVE CARE UNIT IN SFAX-TUNISIA}

Amel Ben Hmed, Mouna Loukil, Chiraz Regaieg*, Manel Charfi, Amira Bouraoui, Nedia Hmida, Ridha Regaieg, Afef Ben Thabet, Abdellatif Gargouri. Department of Neonatology, Hedi Chaker Hospital, Sfax, Tunisia

\subsection{6/archdischild-2019-epa.963}

Background In view of the multiplicity of places of birth and the scarcity of neonatal intensive care units, the transfer of sick newborns is necessary in several situations in order to provide them with the necessary care not available at the birth places. This transport exposes the newborn to some risks that may affect morbidity and mortality.

Aims of the study To study the characteristics of transported newborns and the conditions of their transfer in order to identify the factors that can influence morbidity and mortality.

Methods Retrospective chart review: the medical records of 273 newborns transported and admitted in NICU over a period of 3 years, from January 1, 2012 to December 31, 2014.

Results The transfer rate during the study period was $6.4 \%$. The private sector contributed in $57.5 \%$ of the transfers while the rest of the cases were originated from the peripheral maternities $(25.2 \%)$. The main reasons for transfer were neonatal respiratory distress (62.3\%), neonatal infection (43.2\%) and prematurity (24.2\%).Surgical diseases (or other malformations) were addressed mainly from peripheral maternities with a significant difference compared to the private sector. $89.4 \%$ of cases were transported in an ambulance with a very limited equipment (incubator, oxygen cylinder, rarely a scope) and in the presence of a single nurse and exceptionally a doctor. Incidents occurred during the transfer and were observed in 6 cases: accidental extubation, depletion of the oxygen cylinder. Complications were observed in 48.4 of the cases, the main one was iatrogenic infection (19.75\%). The death rate was $13.6 \%$ : this rate is influenced by hypoglycaemia, hypoxia, surgical diseases and the use of mechanical ventilation.
Conclusion The severity of the initial neonatal pathology conditionned the prognosis of the newborns transported which is also influenced by the conditions of the transport.

\section{P633 NON-CATHETER-RELATED NEONATAL AORTIC THROMBOSIS REVEALING AN ASSOCIATION OF FACTOR $\checkmark$ LEIDEN AND HYPERHOMOCYSTEINEMIA}

Ben Thabet Afef, Manel Charfi*, Amel Ben Hamad, Chiraz Regaieg, Ridha Regaieg, Nedia Hmida, Amira Bouraoui, Abdellatif Garguri. Department of Neonatology, Hedi Chaker University Hospital, Sfax, Tunisia

\subsection{6/archdischild-2019-epa.964}

Introduction Non-catheter-related aortic thrombosis is a rare condition in neonates. It may be life threatening or leads to severe complications. It occurrence requires looking for an underlying congenital prothrombotic condition.

Methods We report a case of a spontaneous aortic thrombosis in a newborn revealing an association of factor $\mathrm{V}$ Leiden and hyperhomocysteinemia.

Results A full term male was born by c-section. He presented immediate severe respiratory distress. Echocardiography performed at the second day of life, showed persistent pulmonary hypertension. He required high frequency oscillatory ventilation and inhaled monoxide administration. Initial respiratory stabilization was noted. But at 11 days age, we noted an increase in oxygen requirement; a tachycardia, a hepatomegaly, an edema and femoral pulses were no more detected. Control echocardiography showed a left ventricular dysfunction with an ejection fraction of $30 \%$. The abdominal doppler ultrasound found an extensive thrombosis in infrarenal abdominal aorta. Thrombolytic treatment was not administrated as a subarachnoid hemorrhage was found in the cerebral ultrasound. After 48 hours of mechanical ventilation and inotropic support, hemodynamic and respiratory stabilization was obtained. Control echocardiography at day 14 , showed an ejection fraction of 50\%. The biological assessment revealed heterozygosis R506Q mutation for the factor V (factor V Leiden) and heterozygosis MTHFR C677T mutation (hyperhomocysteinemia). The aortic thrombosis has been spontaneously lysed and disappeared within 10 months. At 4 years, his physical examination is normal.

Conclusions Till now, there are no clear establisched guidelines concerning the management of arterial thrombosis. We insist that the blood clotting screen must be systematic and complete to look for association of congenital prothrombotic conditions which would increase the thrombotic risk.

\section{P634 HOSPITAL MORTALITY OF FULL-TERM NEWBORNS: FACTORS AND CAUSES}

Amel Ben Hamed, Ilyes Regaieg, Mouna Loukil, Manel Charfi, Chiraz Regaieg*, Amira Bouraoui, Ridha Regaieg, Nedia Hmida, Afef Ben Thabet, Abdelatif Gargouri. Department of Neonatology, Hedi Chaker Hospital, Sfax, Tunisia

\subsection{6/archdischild-2019-epa.965}

Introduction Full-term newborns mortality's is a public health issue because of its unexpected impact either on the infant's family and the medical staff, as opposed to the preterm death. Therefore, we aim to determine factors and causes associated with hospital mortality of full-term newborns. 
Methods This is a retrospective and descriptive study including all full-term neonates deceased before leaving hospital. The Data were collected between January 2016 and December 2018 from the neonatology department of Sfax's University Hospital HédiChaker (Tunisia).

The International Classification of Diseases (ICD) codes' have been followed to classify death's diagnosis.

Results During the study's period 93 term newborns had died in the hospital. $68 \%$ were born by caesarean section and $24 \%$ had a low birth weight. The death occurred during the first week of life in $74 \%$ of cases. The leading causes of death were congenital malformations (56\%) and perinatal asphyxia (22\%). Only 2 cases were deceased by other causes than maternofetal complications.

Conclusion The prenatal diagnosis of fetal anomalies and the quality of the childbirth monitoring are the main factors associated to the hospital term neonatal death. Further research is required to understand causes of full-term's mortality so its incidence and effects can be reduced.

\section{P635 IMPACT OF ANTENATAL CORTICOSTEROID THERAPY ON THE NEONATAL PROGNOSIS OF PRETERM INFANTS BORN BEFORE 33 WEEKS OF AMENORRHEA}

Ilyes Regaieg, Amel Ben hmed, Manel Charfi, Chiraz Regaieg*, Amira Bouraoui, Nedia Hmida, Ridha Regaieg, Afef Ben Thabet, Abdellatif Gargouri. Department of Neonatology, Hedi Chaker Hospital, Sfax, Tunisia

\subsection{6/archdischild-2019-epa.966}

Introduction Antenatal corticosteroid treatment is widely proved to be efficient in reducing hyaline membrane disease and prematurity complications. However, the effects of administering a course of corticosteroids to the mother prior to anticipated preterm birth on fetal and neonatal morbidity and mortality are still unclear. Therefore, the objective of this researchis to assess theses effects on preterm infants born before 33 weeks of amenorrhea.

Patients and methods In this retrospective and descriptive study, we have considered all premature infants who were born before 33 weeks and hospitalized in the neonatology department of Sfax's University Hospital Hedi Chaker (Tunisia) between 1 January 2014 and 31 December 2016.

The short-term prognosis was compared for preterm newborns according to whether antenatal corticotherapy was performed or not.

Results During the period of research, 565 premature infants born before 33 weeks of amenorrhea were hospitalized. Thus the rate of the early prematurity was $1.9 \%$.

Antenatal corticosteroids were performed in $76.1 \%$ of cases.

The frequency of hyaline membrane disease was $22.8 \%$ in the presence of this corticosteroid treatment against $44.4 \%$ when it was not performed.

The incidence of intracranial haemorrhage and ulcerative necrotizing enterocolitis was higher in premature infants who did not receive antenatal corticosteroid therapy.

The overall hospital mortality was $25.1 \%$ in this series. It was significantly higher in the absence of antenatal corticosteroid therapy (39.3\% versus $20.7 \%)$.
The main causes of death were immaturity, hyaline membrane disease, nosocomial infections and intracranial haemorrhage.

Conclusion The antenatal corticosteroid therapy has a great impact on mortality of preterm infants born before 33 weeks. Our findings highlight the value antenatal corticotherapy to reduce morbidity and mortality in these premature infants.

Follow-up studies into childhood and adulthood, particularly in the psychomotor and neuro-development is needed and it would be especially relevant to explore this finding in adequately powered prospective trials.

\section{P636 AN UNUSUAL CAUSE OF GLUTEAL SWELLING.... CONGENITAL RECTAL DUPLICATION CYST}

${ }^{1}$ Qasim Mahmood, 'Uzair Shabbir*, ${ }^{2}$ Brian Sweeney, ${ }^{2}$ Maureen O'Sullivan, ${ }^{1} T a h a$ Yousif. ${ }^{1}$ University Hospital Kerry, Tralee, Ireland; 'Our Lady's Children Hospital, Crumlin, Dublin, Ireland

\subsection{6/archdischild-2019-epa.967}

Rectal Duplication (RD) is a very rare phenomenon with only around 100 cases reported.They make up a merely 5\% of all intestinal duplication's. Most of the rectal duplication's are located in the retro rectal space.

Presenting symptoms include intestinal obstruction,bladder outlet obstruction,dysuria,pelvic pain,mucous or purulent drainage from the rectum or perianal fistula,rectal bleeding from the presence of heterotopic gastric mucosa,constipation and rectal prolapse. RDs are mostly diagnosed with ultrasound and MRI.Treatment of choice in majority of cases is surgical resection.

We report a very unusual case of congenital rectal duplication cyst in a newborn.A male baby was delivered by emergency C-section for maternal pre-eclampsia at 39 weeks gestation with birth weight of $3.9 \mathrm{~kg}$.Initial clinical review revealed a large,shiny,dark bluish cystic type lesion on the right buttock.Ultrasound scan of the right buttock showed a large deep cystic lesion measuring $3 \mathrm{~cm}$ in diameter in the right gluteal region.At 16 hours of age,the lesion burst through one centimeter opening discharging copious amount of liquid meconium,resulting in the collapse of the swelling.As a result,he received fluid resuscitation,feeds were withheld and he was started on antibiotics for suspected sepsis.

MRI pelvis showed a large fluid filled tubular structure in the presacral space displacing both the rectum and bladder. The imaging also showed dilatation of the upper tracts in relation to both kidneys with displaced and distended bladder. Surgery showed rectal duplication cyst sharing a common wall with the rectum,however,there was no direct connection with the rectal lumen and was successfully excised.

The biopsies revealed degenerative and necrotic tissue which was unusual.The presence of meconium could be attributed to the presence of a direct connection with the rectal cavity at some stage of the development.

The baby was discharged home post surgery and made full recovery without any complications.

To our knowledge,this case is the first to present with gluteal swelling and subsequent rupture yielding liquid meconium. 